\section{Conocimientos, actitudes y prácticas de normas de bioseguridad y riesgo biológico en odontólogos de práctica privada de tres ciudades de Nicaragua}

\author{
Knowledge, attitudes and practices of \\ biosecurity and biological risk standards in \\ private dentists of three cities of Nicaragua
}

ISSN: 1560-9111

\section{Artículo Original}

\author{
Manuel Amed Paz Betanco 1,a \\ 1 Universidad Nacional Autónoma de Nicaragua. \\ León, Nicaragua. \\ ${ }^{a}$ Máster en Salud Pública. \\ Correspondencia: \\ Manuel Amed Paz Betanco \\ Correo electrónico: \\ manuel.paz@fo.unanleon.edu.ni \\ Complejo Docente de la Salud (UNAN-León). \\ Edificio Principal 3er. Piso. Ala Sur-este. Depar- \\ tamento de Medicina Oral. Apartado Postal 68. \\ Nicaragua.
}

\section{Conflicto de intereses: No hay conflicto de intereses. \\ Fuente de financiamiento: Autofinanciado.}

Fecha de recepción: 01/11/18

Fecha de aceptación: 21/12/18

\begin{abstract}
Resumen
Objetivo: Describir los conocimientos, actitudes y prácticas de normas de bioseguridad y riesgo biológico en odontólogos que ejercen su práctica privada en tres ciudades de Nicaragua, durante el año 2017. Métodos: Se realizó un estudio descriptivo transversal con 86 dentistas seleccionados con la técnica bola de nieve a través de un muestreo no probabilístico y por conveniencia en las ciudades de Managua,Chinandega y León; a los cuales se le aplicó un cuestionario semiestructurado previamente validado. Los datos fueron procesados utilizando estadística descriptiva mediante frecuencias y porcentajes de las variables en estudio. Resultados: Los odontólogos conocen el concepto de riesgo biológico en un $98,8 \%$, pero no el nombre específico de los agentes infecciosos y las vías de entrada de éstos al organismo (76,7\%), realizan el lavado de manos frecuente antes y después de cada procedimiento dental $(60,4 \%)$ y tienen nociones básicas de actuación ante accidentes percutáneos en un $48,8 \%$, la mayoría responde a buenas actitudes de desinfección y esterilización de instrumentales y equipos $78 \%$ y $68,6 \%$, así como bioseguridad personal (98,8\%). La ciudad de Managua resalta por el adecuado manejo de residuos bioinfecciosos. Conclusiones: Los dentistas presentaron un conocimiento regular, una actitud positiva y buenas prácticas de bioseguridad.
\end{abstract}

Palabras clave: Conocimientos, actitudes y práctica en salud; Bioseguridad; Odontología; Nicaragua. 


\begin{abstract}
Objective: Describe the knowledge and performance of biosecurity and biological risk standards of dentists in private practices from three cities of Nicaragua; during the year 2017. Methods: A transversal descriptive study was implemented on 86 dentists selected with the snowball technique through a non-probabilistic sampling and for convenience, in the cities of Managua, Chinandega and León; a previously validated semi-structured questionnaire was applied. The data was processed using descriptive statistics through frequencies and percentages of the variables under study. Results: The dentists know the concept of biological risk in $98.8 \%$, but not the specific name of the infectious agents and their entrance pathway into the host $(76.7 \%)$, however, they perform frequent hand washing before and after each dental procedure (60.4\%) and have basic notions of the protocol to follow when percutaneous accidents happen in $48.8 \%$; the majority demonstrated good performance during disinfection and sterilization of instruments and equipment $78 \%$ and $68.6 \%$, as well as personal biosecurity (98.8\%). The city of Managua stands out for the adequate management of bio-infectious waste. Conclusions: Dentists presented a regular knowledge, fair performance and good biosecurity practices. Keywords: Health knowledge, attitudes, practice; Biosafety; Dentistry; Nicaragua.
\end{abstract}

\section{Introducción}

Ardila y Muñoz ${ }^{1}$ hacen referencia que diversos estudios en áreas de la salud y seguridad en el trabajo a nivel mundial, demuestran que la exposición laboral a infecciones agudas o crónicas causadas por diversos agentes, especialmente virus, hongos y bacterias, en áreas como: urgencias, quirófanos, odontología, laboratorios clínicos, entre otros, son factores de riesgo para la salud del trabajador y de la comunidad.

De acuerdo con Ayón $e t$ al. ${ }^{2}$ los profesionales y estudiantes de odontología están expuestos a adquirir infecciones provenientes principalmente de la sangre y saliva de los pacientes, debido al manejo de material punzocortante, instrumentos rotatorios y al aerosol generado durante la atención, en un campo restringido de visualización y sujeto al movimiento del paciente. El riesgo biológico depende de factores como el volumen y la naturaleza del fluido, la patogenicidad del agente infeccioso, las condiciones clínicas del paciente y de la persona expuesta, y las medidas seguidas después de la exposición.

En un estudio realizado el 2011 por Aguilera et al. ${ }^{3}$ con dentistas de la ciudad de Chinandega, Nicaragua y sus municipios, sobre los conocimientos, actitudes y prácticas del uso de barreras de protección en relación a enfermedades infectocontagiosas, observaron que tenían un buen conocimiento, la actitud fue positiva, el uso de las barreras de protección en los pacientes eran adecuados, los instrumentos por lo general eran esterilizados en autoclave y los desechos odontológicos los depositaban en recipientes especiales para su eliminación en la basura municipal.

Dado que el odontólogo ejerce una profesión de alto riesgo ${ }^{4}$ es de vital importancia la evaluación periódica en materia de bioseguridad para brindar una atención dental con calidad, por lo que se consideró necesario, describir los conocimientos, actitudes y prácticas de normas de bioseguridad en odontólogos de práctica privada de tres ciudades de Nicaragua durante el periodo agosto-noviembre del año 2017.

\section{Métodos}

Se realizó un estudio descriptivo, transversal; la población estuvo conformada por 86 odontólogos de práctica privada que ejercían en tres ciudades del pacífico nicaragüense: Chinandega, León y Managua. Las clínicas o consultorios odontológicos privados fueron localizados a través de la guía telefónica, publicidad en revistas de cine o asociadas al sector médico, Facebook, Google maps, y con la técnica bola de nieve seleccionados por medio de un muestreo no probabilístico. Se llevó a cabo, de esta manera, la búsqueda de los sujetos de estudio debido a que, en Nicaragua, no hay un registro nacional sobre la ubicación de las clínicas dentales por ciudad y en el Colegio Odontológico o en Asociaciones Odontológicas locales no son muchos los dentistas agremiados. Se incluyeron a todos los cirujanos dentistas de las tres ciudades que aceptaron participar en el estudio y se excluyeron aquellos profesionales con labor docente dentro de la carrera de odontología y que ejercieran su práctica únicamente en el sector público.

Por lo que la población quedó constituida con el $65 \%$ de las ciudades del occidente del país -Chinandega y León-y el 35\% de la capital Managua.

La fiabilidad del cuestionario obtuvo un alfa de Cronbach de $0,86\left(\mathrm{IC}_{95 \%} 0,81-0,94\right)$, de esta manera, quedó integrado con 18 preguntas: tres en el rubro de conocimientos, cinco en el de actitudes y 10 sobre prácticas de normas de bioseguridad.

Los parámetros que se tomaron en cuenta para clasificar los conocimientos, las actitudes y las prácticas fueron considerados de acuerdo a la escala de Likert de la siguiente manera: conocimientos y prácticas buenas si las respuestas correctas correspondían del 70-100\%, regular del 50-70\% y malo del $49 \%$ o menos. Las actitudes fueron considerados positivas si las respuestas correctas eran mayor al $60 \%$ y negativas si el resultado encontrado era menor o igual al $60 \%$.

La presente investigación no pasó por un Comité de Ética Odontológica debido a que la Facultad de Odontolo- 
gía de la UNAN-León no cuenta con dicha estructura, todavía se está trabajando en su creación y funcionamiento, para cumplir los aspectos éticos en la investigacion la encuesta contó con una hoja de consentimiento informado donde a cada participante se le aseguró sigilo profesional, uso de la información con fines investigativos y de planificación de programas de educación continua en materia de bioseguridad en la Facultad de Odontología de la Bicentenaria Universidad Nacional Autónoma de Nicaragua, León (UNAN-León).

Los datos fueron recolectados por un equipo de cuatro estudiantes de la carrera de odontología de la UNANLeón dirigidos por el académico a cargo de la línea de investigación, los cuales fueron previamente entrenados y calibrados para recolectar las encuestas. Ningún estudiante recibió aporte económico por el trabajo realizado debido a que fue parte de su preparación en investigación científica odontológica. Los datos recolectados fueron analizados en el paquete estadístico SPSS versión 21 , con el que se obtuvieron frecuencias y porcentajes de las variables de estudio.

\section{Resultados}

Del total de los dentistas entrevistados, el 1,2\% (1) no conocía el concepto de riesgo biológico; el 76,7\% (66) no conocía el nombre específico de los agentes biológicos con capacidad infecciosa en la clínica dental. Aún cuando más del 50\% mencionó las vías de entrada de los agentes biológicos en odontología, el 44,2\% expresó otro tipo de vías de contagio (Tabla 1).

Se observó que el 48,8\% (42) de los dentistas tienen nociones básicas y fundamentales sobre cómo actuar ante un accidente ocupacional, el 60,4\% (52) de los en- cuestados mencionó el lavado de manos como un hábito frecuente en la atención a los pacientes (Tabla 2).

Se encontró que solo el 19,8\% (17) de los dentistas encuestados desinfecta las mangueras de succión de la unidad dental una vez a la semana, el 43,0\% (37) desinfecta las mangueras del módulo antes de iniciar la jornada de trabajo y posterior a ésta y el 78\% (67) aisla cada superficie del sillón dental posterior a la desinfección de la unidad dental (Tabla 3).

El 68,6\% (59) de los dentistas, después del lavado del instrumental lo deposita en algún tipo de sustancia química. El 18,6\% (16) mencionaron usar el glutaraldehído y dentro de las frecuencias más altas un 7\%(6) expresaron utilizar el Alkazyme (Tabla 4).

El 98,8\% (85) usa todas las barreras de protección física como operador y en los pacientes el 22,1\% (19) de dentistas de León mencionan una lista de barreras de protección que usan en sus pacientes, mientras tanto el 40,7\% (35) de los dentistas de Chinandega y Managua solo mencionaron utilizar los baberos y lentes protectores para proteger a sus pacientes (Tabla 5).

El 89,5\% (77) de dentistas utiliza contenedores rígidos para el material cortopunzante. Es importante también destacar que el $67,4 \%$ (58) de profesionales cuenta con un sitio de almacenamiento de los desechos sólidos. Además, el 63,9\% (55) de los clínicos usan algún sistema de clasificación de los residuos sólidos (Tabla 6).

En el contexto anterior se puede considerar que, los conocimientos sobre bioseguridad que tienen los dentistas son regulares; las actitudes preventivas, positivas y las prácticas de normas de bioseguridad, buenas (Tabla 7).

Tabla 1. Conocimientos de los dentistas sobre riesgo biológico

\begin{tabular}{|c|c|c|c|c|c|c|c|c|c|}
\hline \multirow{2}{*}{ Pregunta } & \multirow{2}{*}{ Respuesta } & \multicolumn{2}{|c|}{ Chinandega } & \multicolumn{2}{|c|}{ León } & \multicolumn{2}{|c|}{ Managua } & \multicolumn{2}{|c|}{ Total } \\
\hline & & $\mathbf{n}$ & $\%$ & $\mathbf{n}$ & $\%$ & $\mathbf{n}$ & $\%$ & $\mathbf{n}$ & $\%$ \\
\hline Conoce el concepto de riesgo biológico. & $\mathrm{Si}$ & 30 & 34,9 & 26 & 30,2 & 29 & 33,7 & 85 & 98,8 \\
\hline $\begin{array}{l}\text { ¿Cuáles son los agentes biológicos con capa- } \\
\text { cidad infecciosa en el consultorio dental? }\end{array}$ & No sabe un nombre específico & 26 & 39,4 & 14 & 16,3 & 26 & 30,2 & 66 & 76,7 \\
\hline \multirow{2}{*}{$\begin{array}{l}\text { ¿Cuál cree es el agente biológico de mayor } \\
\text { peligrosidad de transmisión en la atención } \\
\text { odontológica? }\end{array}$} & VIH/SIDA & 17 & 19,8 & 11 & 12,8 & & & 28 & 32,5 \\
\hline & Virus de la hepatitis B & & & & & 12 & 13,9 & 12 & 13,9 \\
\hline
\end{tabular}

Tabla 2. Actitud del dentista ante el lavado de manos y los accidentes ocupacionales en la atención odontológica

\begin{tabular}{|c|c|c|c|c|c|c|c|c|c|}
\hline \multirow{2}{*}{ Pregunta } & \multirow{2}{*}{ Respuesta } & \multicolumn{2}{|c|}{ Chinandega } & \multicolumn{2}{|c|}{ León } & \multicolumn{2}{|c|}{ Managua } & \multicolumn{2}{|c|}{ Total } \\
\hline & & $\mathbf{n}$ & $\%$ & $\mathbf{n}$ & $\%$ & $\mathbf{n}$ & $\%$ & $\mathbf{n}$ & $\%$ \\
\hline \multirow[b]{2}{*}{$\begin{array}{l}\text { Frecuencia del lavado } \\
\text { de manos }\end{array}$} & Antes y después de cada procedimiento & 18 & 20,9 & & & 21 & 24,4 & 39 & 45,3 \\
\hline & $\begin{array}{l}\text { Después de cada procedimiento, luego de usar el } \\
\text { celular y después de tocar una superficie en la clínica } \\
\text { dental }\end{array}$ & & & 13 & 15,1 & & & 13 & 15,1 \\
\hline \multirow{2}{*}{$\begin{array}{l}\text { ¿Qué haría en caso de } \\
\text { sufrir un accidente } \\
\text { en un procedimiento } \\
\text { dental y pincharse o } \\
\text { herirse? }\end{array}$} & $\begin{array}{l}\text { Retirarse los guantes, lavar la herida con jabón } \\
\text { germicida y colocarse una torunda de algodón con } \\
\text { alcohol por } 3 \text { minutos y visitar la Unidad de Salud } \\
\text { más cercana comunicando que sufrí un accidente } \\
\text { laboral. }\end{array}$ & 17 & 19,8 & 14 & 16,3 & & & 31 & 36,0 \\
\hline & $\begin{array}{l}\text { Retirarse los guantes, neutralizo el sangrado con } \\
\text { alcohol, me coloco nuevos guantes y continuo con el } \\
\text { procedimiento. }\end{array}$ & & & & & 11 & 12,8 & 11 & 12,8 \\
\hline
\end{tabular}


Tabla 3. Actitud del dentista ante la desinfección de la unidad dental

\begin{tabular}{|c|c|c|c|c|c|c|c|c|c|}
\hline \multirow{2}{*}{ Pregunta } & \multirow{2}{*}{ Respuesta } & \multicolumn{2}{|c|}{ Chinandega } & \multicolumn{2}{|c|}{ León } & \multicolumn{2}{|c|}{ Managua } & \multicolumn{2}{|c|}{ Total } \\
\hline & & $\mathbf{n}$ & $\%$ & $\mathrm{n}$ & $\%$ & $\mathrm{n}$ & $\%$ & $\mathrm{n}$ & $\%$ \\
\hline $\begin{array}{l}\text { ¿Cada cuánto desinfecta las mangue- } \\
\text { ras de succión de la unidad dental? }\end{array}$ & Una vez a la semana & 7 & 8,1 & 5 & 5,8 & 5 & 5,8 & 17 & 19,8 \\
\hline $\begin{array}{l}\text { ¿Cada cuánto desinfecta las mangue- } \\
\text { ras del módulo? }\end{array}$ & $\begin{array}{l}\text { Antes de iniciar la jornada } \\
\text { de trabajo y posterior a ésta }\end{array}$ & 11 & 12,8 & 9 & 10,4 & 17 & 19,8 & 37 & 43,0 \\
\hline $\begin{array}{l}\text { Posterior a la desinfección: ¿aísla cada } \\
\text { superficie de contacto del sillón? }\end{array}$ & $\mathrm{Si}$ & 17 & 19,8 & 22 & 25,6 & 28 & 32,5 & 67 & 78 \\
\hline
\end{tabular}

Tabla 4. Prácticas de normas de bioseguridad en materia de desinfección y esterilización

\begin{tabular}{|c|c|c|c|c|c|c|c|c|c|}
\hline \multirow{2}{*}{ Pregunta } & \multirow{2}{*}{ Respuesta } & \multicolumn{2}{|c|}{ Chinandega } & \multicolumn{2}{|c|}{ León } & \multicolumn{2}{|c|}{ Managua } & \multicolumn{2}{|c|}{ Total } \\
\hline & & $\mathbf{n}$ & $\%$ & $\mathbf{n}$ & $\%$ & $\mathrm{n}$ & $\%$ & $\mathrm{n}$ & $\%$ \\
\hline $\begin{array}{l}\text { ¿Después que lava el instrumental } \\
\text { lo deposita en alguna sustancia } \\
\text { química? }\end{array}$ & $\mathrm{Si}$ & 17 & 19,8 & 17 & 19,8 & 25 & 29,1 & 59 & 68,6 \\
\hline \multirow{2}{*}{$\begin{array}{l}\text { ¿Cuál es el nombre de la sustancia } \\
\text { química que usa? }\end{array}$} & Glutaraldehído & 11 & 12,8 & 5 & 5,8 & & & 16 & 18,6 \\
\hline & $\begin{array}{l}\text { Alkazyme } \\
\text { (Mono-enzimático) }\end{array}$ & & & & & 6 & 7 & 6 & 7 \\
\hline \multirow{2}{*}{$\begin{array}{l}\text { ¿Qué medio usa para la esterilización } \\
\text { del instrumental? }\end{array}$} & Estufa & & & 10 & 11,6 & & & 10 & 11,6 \\
\hline & Autoclave & 20 & 23,2 & & & 22 & 25,6 & 42 & 48,8 \\
\hline $\begin{array}{l}\text { ¿Cómo maneja el instrumental y } \\
\text { equipos no críticos? }\end{array}$ & Sólo desinfecta con Lysol® & 19 & 22,1 & 15 & 17,4 & 14 & 16,3 & 48 & 55,8 \\
\hline
\end{tabular}

Tabla 5. Prácticas de barreras de bioseguridad en el equipo de trabajo dental y pacientes

\begin{tabular}{|c|c|c|c|c|c|c|c|c|c|}
\hline \multirow{2}{*}{ Pregunta } & \multirow{2}{*}{ Respuesta } & \multicolumn{2}{|c|}{ Chinandega } & \multicolumn{2}{|c|}{ León } & \multicolumn{2}{|c|}{ Managua } & \multicolumn{2}{|c|}{ Total } \\
\hline & & $\mathbf{n}$ & $\%$ & $\mathrm{n}$ & $\%$ & n & $\%$ & $\mathrm{n}$ & $\%$ \\
\hline $\begin{array}{l}\text { Usa todas las barreras de pro- } \\
\text { tección física como operador. }\end{array}$ & $\mathrm{Si}$ & 30 & 34,9 & 25 & 29,1 & 30 & 34,9 & 85 & 98,8 \\
\hline \multirow[t]{2}{*}{$\begin{array}{l}\text { ¿Qué barreras usa para la pro- } \\
\text { tección de sus pacientes? }\end{array}$} & $\begin{array}{l}\text { Lentes protectores, baberos, papel } \\
\text { de aluminio/plástico adhesivo en } \\
\text { superficies del sillón, succión, vasos } \\
\text { desechables y servilletas por paciente }\end{array}$ & & & 19 & 22,1 & & & 19 & 22,1 \\
\hline & Baberos y lentes protectores & 18 & 20,9 & & & 17 & 19,8 & 35 & 40,7 \\
\hline
\end{tabular}

Tabla 6. Almacenamiento y eliminación de desechos peligrosos bioinfecciosos

\begin{tabular}{|c|c|c|c|c|c|c|c|c|c|}
\hline \multirow{2}{*}{ Pregunta } & \multirow{2}{*}{ Respuesta } & \multicolumn{2}{|c|}{ Chinandega } & \multicolumn{2}{|c|}{ León } & \multicolumn{2}{|c|}{ Managua } & \multicolumn{2}{|c|}{ Total } \\
\hline & & $\mathbf{n}$ & $\%$ & $\mathrm{n}$ & $\%$ & $\mathbf{n}$ & $\%$ & $\mathrm{n}$ & $\%$ \\
\hline $\begin{array}{l}\text { Usa contenedores rígidos para } \\
\text { el material cortopunzante }\end{array}$ & $\mathrm{Si}$ & 26 & 30,2 & 23 & 26,7 & 28 & 32,5 & 77 & 89,5 \\
\hline $\begin{array}{l}\text { Cuenta con un sitio de alma- } \\
\text { cenamiento de los desechos } \\
\text { sólidos }\end{array}$ & $\mathrm{Si}$ & 22 & 25,6 & 14 & 16,3 & 22 & 25,6 & 58 & 67,4 \\
\hline $\begin{array}{l}\text { Usa algún sistema de clasifica- } \\
\text { ción de los residuos sólidos }\end{array}$ & $\mathrm{Si}$ & 17 & 19,8 & 18 & 20,9 & 20 & 23,2 & 55 & 63,9 \\
\hline \multirow{3}{*}{$\begin{array}{l}\text { Lugar donde almacena los } \\
\text { residuos sólidos }\end{array}$} & $\begin{array}{l}\text { Tengo un contenedor con tapadera fuera } \\
\text { del consultorio }\end{array}$ & 13 & 15,1 & & & & & 13 & 15,1 \\
\hline & $\begin{array}{l}\text { No tengo un sitio específico, los almace- } \\
\text { no junto con la basura común del hogar }\end{array}$ & & & 13 & 15,1 & & & 13 & 15,1 \\
\hline & $\begin{array}{l}\text { Hay una compañía de bioseguridad } \\
\text { hospitalaria que se encarga de todo el } \\
\text { proceso. }\end{array}$ & & & & & 14 & 16,3 & 14 & 16,3 \\
\hline
\end{tabular}

Tabla 7. Conocimientos, actitudes y prácticas de los odontólogos

\begin{tabular}{lc}
\hline Consolidado & Resultado \\
\hline Conocimiento sobre riesgo biológico & Regular \\
Actitud preventiva & Positiva \\
Práctica de normas de bioseguridad & Buena \\
\hline
\end{tabular}




\section{Discusión}

A pesar que en Nicaragua la profesión odontológica no es regulada por una entidad gubernamental ni por una asociación odontológica colegiada, los resultados obtenidos en este estudio, permiten una mirada descriptiva de los dentistas de práctica privada que atienden a la mayor parte de la población del país, debido a que en el sector público solo se realizan exodoncias y destartraje periodontal. Por la variedad de tratamientos dentales que se ofertan en la práctica privada es importante resaltar que, a pesar no estar bajo control constante por una autoridad supervisora tienen una actitud positiva ante la prevención de riesgos biológicos y realizan buenas prácticas de normas de bioseguridad en el gabinete dental.

Los resultados de este estudio muestran por parte de los dentistas, un manejo práctico de todo lo concerniente a la higienización, desinfección y calidez humana al brindar la atención dental, no solo con los protocolos aprendidos durante su estancia en la universidad sino que también utilizan lineamientos de atención dental de otros países y con reglamentos específicos para el sector dental de lo que carece el gremio odontológico en Nicaragua.

En esta investigación no se encontró relación entre conocimiento, prácticas y actitudes preventivas, lo que coincide con Núnez et al. ${ }^{5}$ que estudiaron una muestra de estudiantes de odontología de una universidad privada peruana, donde no encontraron asociación estadística entre la actitud, el grado de conocimiento de los estudiantes y esterilización de las piezas de alta y baja velocidad.

En el estudio de Ruiz ${ }^{6}$ acerca de los conocimientos de bioseguridad en el personal de salud de un hospital peruano, se relacionó los conocimientos de bioseguridad con el sexo, no encontrandose diferencias estadísticamente significativas entre éstas variables.

Licea $e t$ al. ${ }^{7}$ encontraron una relación significativa entre las variables conocimiento sobre bioseguridad con el cumplimiento de estas medidas y observaron que a medida que el profesional cuenta con más conocimiento ponen en práctica las normas de bioseguridad. Hernández et al. ${ }^{8}$ encontraron asociación estadísticamente significativa entre el conocimiento que tiene la población estudiada con respecto al protocolo que hay que seguir en caso de un accidente biológico, resultados que no coinciden con nuestro estudio.

Alata y Ramos ${ }^{9}$ encontraron una relación estadísticamente significativa entre conocimientos y prácticas de normas de bioseguridad en estudiantes de odontología. Jiménez y Salgado ${ }^{10}$ en un estudio realizado en Cuba encontraron significancia al relacionar la práctica en prevención de enfermedades con los años de graduado del profesional o del personal técnico, en donde el grupo con mayor experiencia laboral tuvo mejor práctica en esta temática, sin embargo, en esta investigación no se realizó el cruce de estas variables debido a que no se perfiló en esa dirección.
En el estudio de Díaz ${ }^{11}$ realizado en una población universitaria de la ciudad de Cali, se mostró que el 37\% de los estudiantes conocía el concepto de riesgo biológico; en la presente investigación el 98,8\% (85) manifestó conocer el concepto de riesgo biológico, sin embargo, el $76,7 \%$ (66) no conoce el nombre específico del agente biológico con capacidad infecciosa en la clínica dental; resultados que son desalentadores porque se puede observar que el odontólogo en su práctica privada se vuelve más técnico que científico. En referencia al binomio virus de la hepatitis $\mathrm{B}$ y VIH, en ambos estudios los entrevistados hacen mención que las anteriores son patologías transmitidas por la exposición de riesgo biológico por lo que son agentes de mayor peligrosidad de transmisión en la consulta dental; el $42 \%$ para Díaz y el 46,4\% (40) para éste estudio. Ramos et al. ${ }^{12}$ mencionan también frecuencias similares a los estudios antes mencionados en referencia a éstos dos agentes de mayor riesgo de transmisión en la consulta dental.

En el lavado de manos, el 20,9\% (18) de los odontólogos de la ciudad de Chinandega y el 24,4\% (21) de Managua refirieron hacerlo antes y después de cada procedimiento dental, éstos resultados son parecidos a los encontrados por Díaz ${ }^{13}$ en los residentes de la especialización en Endodoncia de una universidad chilena, donde el 13,3\% realizaba el lavado de manos antes de atender pacientes en comparación al 26,7\% que lo realizan después de atender pacientes. Harrita et al. ${ }^{14}$ realizaron un estudio en la ciudad hindú de Chennai sobre los conocimientos, actitudes y prácticas de los odontólogos respecto al lavado de manos, donde encontraron un nivel de conocimiento general moderado a adecuado y en el cuestionario realizado es importante resaltar que el $96,8 \%$ de los dentistas cree que hay un nexo importante entre el lavado de manos y el control de infecciones.

Los odontólogos de las ciudades de León y Chinandega expresaron que en caso de accidentes con presencia de heridas percutáneas lo idóneo era seguir el protocolo que recomienda la Organización Mundial de la Salud que es retirarse los guantes, lavar la herida con jabón germicida, colocarse una torunda de algodón con alcohol por tres minutos y visitar la Unidad de Salud más cercana comunicando que sufrió un accidente laboral, datos que coinciden con lo encontrado por Arrieta et al. ${ }^{15}$ en un estudio realizado a estudiantes de odontología de la Universidad de Cartagena en el año 2012, en donde informan que respecto a accidentes laborales e injurias percutáneas, el 56,8\% de los sujetos de estudio seguían de manera correcta el protocolo de bioseguridad. Sin embargo, no coincide con lo encontrado por Saliba et al. ${ }^{16}$ que en un estudio realizado a estudiantes de los últimos años de la carrera de odontología el 85,5\% expresó no tener o tuvieron poca información sobre qué hacer en el caso de accidente con material contaminado.

Una vez a la semana es la respuesta que con mayor frecuencia dieron los odontólogos respecto a la higienización y desinfección de las mangueras del sistema de succión, por lo que es ésta, una de las actitudes negativas del dentista en este apartado. No se encontraron estudios en 
donde hicieran estas correlaciones entre actitud y desinfección, pero, el manual de bioseguridad de Panamá indica que la desinfección de las mangueras del sistema de succión debe ser entre cada paciente y al final de la jornada de trabajo y protegiendo con barreras ${ }^{17}$.

El glutaraldehído es el químico que más usan los dentistas en la desinfección de alto nivel del instrumental crítico y semicríticos antes de ser esterilizados ya sea por calor seco o autoclave, es interesante resaltar en este momento el estudio microbiológico realizado por Gutiérrez et al. ${ }^{18}$ llevado a cabo en las clínicas odontológicas de la Universidad Antonio Nariño sede sur, con tres sustancias químicas, en tres sitios propicios para el desarrollo bacteriano, en el cual se demostró que el que presenta mejor acción antimicrobiana es el glutaraldehído al $2 \%$. Lunelli, et al. ${ }^{19}$ muestran en un grupo de endodoncistas de una ciudad brasileńa que el autoclave es el método utilizado para esterilizar el instrumental endodóntico, y de éstos el 68,7\% usa detergente enzimático, porcentaje alto comparado al encontrado en el presente estudio (7\%), ya que sólo 6 dentistas de la ciudad de Managua expresaron usar detergente monoenzimático antes de esterilizar el instrumental odontológico.

En nuestro estudio, los dentistas reportaron que el instrumental y equipo no crítico del consultorio dental lo desinfectaban con aerosol desinfectante Lysol $^{\circ}$, al respecto, Iturralde ${ }^{20}$ comparó microbiológicamente la desinfección efectuada en las superficies de las jeringas triples de unidades odontológicas con dos marcas de aerosoles Lysol $^{\oplus}$ y Eucida ${ }^{\circledR}$ concluyendo que no había diferencias entre estos aerosoles, ya que el recuento inicial de aerobios disminuyó significativamente con los dos químicos $(\mathrm{p}<0,05)$.

En este estudio los odontólogos informaron que ellos, su equipo de trabajo (dentista-asistente) al igual que con sus pacientes, usan barreras físicas de protección, resultado que coincide con lo encontrado por Aguilera et al. ${ }^{3}$ en un estudio realizado en la ciudad de Chinandega, Nicaragua, en donde los dentistas usaban de manera correcta las barreras de protección en sus pacientes; coincide también con lo reportado por Álvarez y Juna ${ }^{21}$ en un estudio efectuado en odontólogos de la ciudad de Lacatunga en donde se evidenció que los profesionales ponían en práctica sus conocimientos sobre el uso de barreras de bioseguridad facial a la atención de sus pacientes. Ramos et al. ${ }^{12}$ especifican que los estudiantes brasileños de odontología mencionan por barrera de protección el uso de mascarillas, chaleco, guantes y gorro, sin embargo, en cuanto a la utilización de gafas de protección, hubo una diferencia estadísticamente significativa por los estudiantes de odontología de centros de educación superior privado, encontrando más del 90\% de práctica de éstas como método para reducir la transmisión de patógenos.

Es importante resaltar ésta práctica de bioseguridad realizada por el grupo de dentistas evaluados, ya que desde la óptica del paciente, el uso de estas barreras como de otros aditamentos entran en los parámetros de calidad de atención, según lo reportaría Paz ${ }^{22}$ en el año 2016 en su estudio sobre percepción de calidad de atención del paciente que acude a las clínicas multidisciplinarias de la Facultad de Odontología de la UNAN-León. Por su parte Briceńo et al. ${ }^{23}$ en un estudio realizado en estudiantes de una universidad venezolana sobre conocimiento y uso del tapaboca y máscara de amplia cobertura encontraron que el $84 \%$ de los encuestados expresó usar el aditamento, además la mayoría de éstos considera la importancia de su uso para la protección contra el intercambio bacteriano y otros agentes irritantes.

En el apartado sobre la gestión de los residuos sólidos bioinfecciosos en este estudio se encontró que los dentistas cuentan con buenas prácticas de bioseguridad en esta materia y tienen el cuidado de usar contenedores rígidos para el material cortopunzante, en clasificar los residuos de acuerdo a las normas internacionales y en contar con un almacenaje intermedio de estos. Sin embargo, se evidenció un mal manejo respecto al lugar de almacenamiento en las ciudades de Chinandega y León, pero un manejo excelente en la capital Managua; debido a que en ésta última existen empresas ecobiológicas que tratan con los residuos bioinfecciosos de origen médico y que se rigen bajo las normas internacionales en materia de bioseguridad. Aguilera et al. ${ }^{3}$ mencionan que los dentistas manejaban los desechos odontológicos depositándolos en recipientes especiales para su eliminación en la basura municipal. Sin embargo, aún en nuestro medio no contamos con ningún estudio que haya realizado seguimiento a la disposición final de los desechos bioinfecciosos provenientes de hospitales y clínicas médico dentales públicas y privadas que realizan los trabajadores municipales.Autores como Camargo et al. ${ }^{24}$ mencionan que en el manejo de los residuos en las clínicas dentales de la Universidad Santo Tomás, los depósitos guardianes están bien dispuestos y son utilizados de forma correcta por la mayoría de los estudiantes y los residuos son clasificados de manera correcta.

Un estudio realizado por Quinto et al. ${ }^{25}$ con 67 trabajadores de un hospital colombiano sobre conocimientos y prácticas del manejo de los residuos, dejó ver que el peor resultado se presentó en la eliminación de desechos hospitalarios con el 41,8\%.

Ruiz ${ }^{6}$ en su estudio, hace mención que las preguntas de la encuesta acerca del proceder del personal posterior a la exposición a material contaminado, manejo y transporte de las muestras tomadas fueron las que obtuvieron menor frecuencia de respuestas correctas.

Finalmente se puede decir que los dentistas encuestados presentaron, un conocimiento regular; una actitud preventiva positiva y algunas buenas prácticas de bioseguridad.

\section{Referencias bibliográficas}

1. Ardila A, Muñoz A. Bioseguridad con énfasis en contaminantes biológicos en trabajadores de la salud. Ciénc Saúde Coletiva. 2009;14(6):2135-2141.

2. Ayón E, Villanelo M, Bedoya L, González R, Pardo K, Picasso $\mathrm{M}$ et al. Conocimiento y actitudes sobre biosegu- 
ridad en estudiantes de Odontología de una Universidad Peruana. KIRU. 2014;11(1):39-45.

3. Aguilera A, Alemán Y, Alvarado J. Conocimientos, actitudes y prácticas del uso de barreras de protección en los Odontólogos de la ciudad de Chinandega y sus municipios de agosto 2010 a marzo 2011. (Tesis de Licenciatura). León: Facultad de Odontología, Universidad Nacional Autónoma de Nicaragua (UNAN-León); 2011.95 p.

4. Tapias L, Fortich N, Castellanos V. Evaluación de los conocimientos y prácticas de bioseguridad para prevenir los eventos adversos y los accidentes ocupacionales en estudiantes de odontología. CSV. 2013;5(1):87-94.

5. Núñez M, Gutiérrez F. Conocimientos y actitudes de estudiantes de estomatología sobre la esterilización de piezas de mano dentales. Rev Estomatol Hered. 2016;26(4):222-228.

6. Ruiz J. Conocimiento de las medidas de bioseguridad en personal de salud. Horiz Med. 2017;17(4):53-57.

7. Licea Y, Rivero M, Solana L, Pérez K. Nivel de conocimientos y actitud ante el cumplimiento de la bioseguridad en estomatólogos. Rev cien méd La Habana. 2012;18(1):80-90.

8. Hernández A, Montoya J, Simancas M. Conocimientos, prácticas y actitudes sobre bioseguridad en estudiantes de Odontología. Rev Colomb investig odontol. 2012;(3)9:148-157.

9. Alata G, Ramos S. Nivel de conocimiento de los alumnos de la EAP de Odontología y aplicación de las medidas de bioseguridad para reducir el riesgo de contagio de enfermedades en la clínica dental de la UNHEVAL-Huánuco-octubre 2010-febrero 2011. (Tesis de Licenciatura). Huánuco: Facultad de Ciencias Médicas, Universidad Nacional Hermilio Valdizán; 2011. 109 p.

10. Jiménez $S$, Salgado M. Conocimientos y prácticas del personal estomatológico sobre la prevención de enfermedades transmisibles. Marianao. 2015-2016. Rev haban cienc méd. 2017;16(5):808-821.

11. Díaz A. Conocimiento en riesgo biológico y prácticas de bioseguridad en el personal docente de la Facultad de Salud de una Institución de Educación Superior de la ciudad de Cali. (Tesis de Maestría). Santiago de Cali: Facultad de Salud, Universidad del Valle. 2013. 110 p.

12. Ramos S, Feitosa A, da Silva F, Gomez J. Formação em odontologia: o papel das instituiçóes de ensino na prevenção de acidentes com exposição a material biológico. Cienc trab. 2015;17(54):182-187.

13. Díaz V. Grado de aplicación de precauciones estándar durante la atención de pacientes por estudiantes de programa de especialización profesional en Endodoncia (Tesis de Licenciatura). Santiago de Chile: Facultad de Odontología, Universidad de Chile; 2016. 98 p.
14. Harrita S, Dhanranj, Prasad P. Knowledge, attitude and practice of hand sanitation among the dentist in Chennai city. Int J Adv Res. 2017;5(4):1699-1706.

15. Arrieta K, Díaz S, González F. Conocimientos, actitudes y prácticas sobre accidentes ocupacionales en estudiantes de odontología. Rev Cub Salud Pública. 2012;38(4):546552.

16. Saliba C, Martins R, Ísper A, Hidalgo L. Conductas de estudiantes del área de la salud frente a exposición ocupacional a material biológico. Cienc Trab. 2009;11(31):1821.

17. Comité Nacional de Bioseguridad en Salud Bucal. Bioseguridad en la práctica bucodental. Normas técnicas y manual de procedimientos. Panamá; 2006.p.174.

18. Gutiérrez S, Dussán D, Leal S y Sánchez A. Evaluación microbiológica de la desinfección en unidades odontológicas (estudio piloto). Rev Colomb Cienc Quím Farm. 2008;37(2):133-149.

19. Lunelli S, Fontoura T, Berwanger C. Analysis of cleaning, sterilization, storage process and frequency of endodontic instrument replacement of endodontists from the city of Caxias do Sul/RS. RSBO. 2017;14(1):11-15.

20. Iturralde A. Comparación del efecto desinfectante entre Lysol y Eucida en las superficies de las jeringas triples de las unidades odontológicas de la clínica integral del séptimo semestre de la Facultad de Odontología de la Universidad Central del Ecuador (Tesis de Licenciatura). Quito: Facultad de Odontología, Universidad Central del Ecuador; 2015.

21. Álvarez F, Juna C. Conocimiento y prácticas sobre bioseguridad en odontólogos de los centros de salud de Lacatunga. Enferm Inv. 2017;2(2):59-63.

22. Paz M. Percepción de la calidad de atención del paciente que acude a las clínicas multidisciplinarias de la Facultad de Odontología, Universidad Nacional Autónoma de Nicaragua (UNAN-León). Odont Vital.2016; 28:29-32.

23. Briceño E, Romero M, Rondón B, Merino R. Conocimiento y uso del tapaboca y máscara de amplia cobertura, en un grupo de estudiantes de odontología de la Universidad Central de Venezuela. Odous Científica. 2017;18(1):21-28.

24. Camargo J, Vera Y, Sierra M. Uso de implementos y medidas de bioseguridad en las clínicas odontológicas de Bucaramanga de la Universidad Santo Tomás en el segundo semestre del año 2015 (Tesis de Licenciatura). Bucaramanga: Universidad Santo Tomás, Facultad de Odontología; 2016.

25. Quinto Y, Jaramillo L, Cardona J. Conocimientos y prácticas de los trabajadores de un hospital sobre el manejo de residuos hospitalrios, Chocó, Colombia, 2012. MÉD UIS. 2013;26(1):9-20. 
